\title{
Dorn, Leon Konstantin, Die Kontrolle vertraglicher Ungleichgewichte zwischen Unternehmen. Eine rechtsvergleichende Untersuchung des reformierten französischen und des deutschen Vertragsrechts.
}

\author{
Mohr Siebeck Verlag, Tübingen, 2021. XXVII, 438 Seiten. ISBN \\ 978-3-16-160009-8
}

\section{Christian Armbrüster}

Angenommen: 30. April 2021 / Online publiziert: 21. Mai 2021

(C) Der/die Autor(en) 2021

Die Frage, inwieweit das objektive Recht der Privatautonomie im Geschäftsverkehr zwischen Unternehmen Schranken setzt, wird seit langem kontrovers diskutiert. Aus jüngerer Zeit ist insbesondere die breit angelegte Monographie von Wendland zu nennen (Vertragsfreiheit und Vertragsgerechtigkeit, 2019, s. dazu Armbrüster, ZVersWiss 2019, $219 \mathrm{ff}$.). Auch Dorn nimmt sich in seiner flüssig und gut verständlich verfassten Arbeit, einer an der Bucerius Law School in Hamburg entstandenen rechtsvergleichenden Dissertation, der Thematik an. Dabei geht es um zwei verschiedene Themenkreise, nämlich die AGB-Kontrolle (S. 27-249) und die allgemeinen Regeln der $\S \S 134,138$ BGB zur Verbots- und Sittenwidrigkeit (insbesondere: Wucher und wucherähnliche Geschäfte; S. 251-385). Aus versicherungsrechtlicher Sicht steht der erstgenannte Bereich in der Praxis ganz im Vordergrund; er soll daher im Folgenden allein betrachtet werden. Hier geht es im Kern darum, inwieweit die Regeln der $\S \S 305$ ff. BGB zu einer Kontrolle von Allgemeinen Versicherungsbedingungen führen, wenn es sich bei dem Versicherungsnehmer um einen Unternehmer handelt.

De lege lata ist die Frage im Grundsatz folgendermaßen zu beantworten: Im unternehmerischen Geschäftsverkehr (sog. b2b-Bereich) gelten die speziellen Klauselverbote der $\S \S 308,309$ BGB zwar nicht unmittelbar, wohl aber haben sie nach der Rechtsprechung des BGH über die Generalklausel des $§ 307$ BGB in weitem Umfang eine Indizwirkung. Unklar und umstritten ist es, inwiefern das Gebot, dass im b2b-Bereich ,auf die im Handelsverkehr geltenden Gewohnheiten und Gebräuche [...] angemessen Rücksicht zu nehmen“" ist (§ 310 Abs. 1 S. 2 Halbs. 2 BGB), zu einer Beschränkung der Inhaltskontrolle führt. Dorn geht auf beide Themen ein.

Christian Armbrüster $(\bowtie)$

Fachbereich Rechtswissenschaft, Freie Universität Berlin, Van't-Hoff-Str. 8, 14195 Berlin,

Deutschland

E-Mail: c.armbruester@fu-berlin.de 
Die Annahme einer Indizwirkung der speziellen Klauselverbote für die Anwendung von $\S 307$ BGB sieht er zu Recht kritisch (S. 195 ff., 203). Wichtiger ist aus versicherungswissenschaftlicher Sicht allerdings die zweite Frage, da bei Allgemeinen Versicherungsbedingungen die Inhaltskontrolle anhand der Generalklausel ganz im Vordergrund steht. Dazu bietet der Autor zunächst zum deutschen Recht eine vergleichsweise breite Darstellung der allgemeinen AGB-rechtlichen Vorgaben (S. 105 ff.), bevor er im Kontext des „Aushandelns“ auf die Besonderheiten bei b2bVerträgen zu sprechen kommt (S. $115 \mathrm{ff}$.).

Dabei erwähnt er das Rücksichtnahmegebot des $§ 310$ Abs. 1 S. 2 Halbs. 2 BGB an verschiedener Stelle; er sieht darin zu Recht ein „Differenzierungsgebot“ (S. 217), ohne allerdings dessen Bedeutung und Reichweite bei der Anwendung der Generalklausel näher zu beleuchten. Hierzu wäre es interessant gewesen zu erfahren, wie der Autor sich zu den von Wendland in der oben genannten Monographie vorgetragenen Thesen positioniert. Auch einige weitere im Schrifttum bereits vor dem im Vorwort als Bearbeitungsstand angegebenen Zeitpunkt (Oktober 2019) veröffentlichte Werke bleiben unberücksichtigt (etwa die Dissertation von Axer, Rechtfertigung und Reichweite der AGB-Kontrolle im unternehmerischen Geschäftsverkehr, 2012; s. zudem die Nachw. bei Armbrüster, NZA-Beil. 2019, $44 \mathrm{ff}$.).

Nur teilweise thematisiert der Autor die zahlreichen Reformvorschläge zum deutschen Recht, die allesamt auf eine Begrenzung der AGB-Kontrolle im b2b-Bereich ausgerichtet sind (s. dazu insbesondere den Gesetzgebungsvorschlag der Frankfurter Initiative, https://recht.vdma.org/documents/14969694/26921572/AGB-Initiative_ Positionspapier_06_2018.pdf/97b30b6a-4778-1f38-d90b-3870dd9ae297?t=370892. 58 (Abruf am 21.04.2021); s. auch Herresthal [Hrsg.], Reform der AGB-Kontrolle im B2B-Bereich, 2020; krit. zu vielen der Vorschläge freilich Graf v. Westphalen, ZIP 2018, 1101, 1107 ff.). Immerhin sieht auch der Koalitionsvertrag zwischen CDU, CSU und SPD für die 19. Legislaturperiode https://www.bundestag.de/resource/blob/ 543200/9f9f21a92a618c77aa330f00ed21e308/kw49_koalition_koalitionsvertragdata.pdf (Abruf am 21.04.2021) Handlungsbedarf; demnach will die Koalition das AGB-Recht für Unternehmen ,,auf den Prüfstand stellen“ (S. 131 des Koalitionsvertrags), um innovativen Geschäftsmodellen mehr Rechtssicherheit zu bieten (vom Verf. einleitend erwähnt auf S. 6). Soweit die Lösung in einer Abkehr vom strengen Verständnis des Begriffs „Aushandeln“ durch den BGH gesucht wird, geht Dorn darauf intensiv ein (S. $120 \mathrm{ff}$.), wobei er sich im Ergebnis für eine großzügigere Auslegung ausspricht. Demnach soll es entscheidend auf die freie und bewusste Entscheidung ankommen, nachdem „faktische Vertragsverhandlungen“ vorausgegangen sind (S. 139).

$\mathrm{Ob}$ sich mit diesem vergleichsweise zurückhaltenden Vorschlag der Vorwurf einer übermäßigen Beschränkung der Privatautonomie ausräumen lässt, mag man durchaus bezweifeln. Die Umsetzung des einen oder anderen der im deutschen Schrifttum diskutierten, weiter reichenden Reformvorschläge würde zwar die vom Autor aufgezeigte, insbesondere durch die große französische Vertragsrechtsreform von 2016 (zu ihr sehr instruktiv S. $24 \mathrm{ff}$., $49 \mathrm{ff}$.) - wodurch die in Deutschland bereits mit dem AGBG von 1976 vollzogene Erstreckung der AGB-Kontrolle auf den b2b-Bereich in Frankreich kodifiziert wurde - erreichte Annäherung des deutschen und des französischen Rechts de lege ferenda nicht unbedingt weiter voranbringen. Was eine 
solche Konvergenz angeht, und zwar diesmal womöglich in Richtung einer Angleichung des deutschen an das (insoweit liberalere) französische AGB-Recht, betreffen die diesbezüglichen Ausführungen (S. 219 ff., zusammenfassend S. 248-249) insbesondere die Frage, ob es für die unangemessene Benachteiligung auf die einzelne Klausel ankommt und eine Kompensation nur bei sog. Zweckkongruenz möglich ist oder ob - wie nach dem französischen Recht - auch ein weiter verstandener Zusammenhang genügt. Die grundlegendere Frage, inwiefern eine Klauselkontrolle im b2b-Bereich überhaupt sachgerecht erscheint, dürfte die Praxis derzeit auch jenseits des Versicherungssektors weitaus stärker bewegen. Hierzu hätte nicht zuletzt interessiert, wie sich die französische Diskussion entwickelt, nachdem seit der Erstreckung der AGB-Kontrolle auf den unternehmerischen Geschäftsverkehr bereits erste Erfahrungen gesammelt werden konnten. Aus solchen Erkenntnissen könnte künftig eine gemeinsame Entwicklung hin zu einer Liberalisierung des b2b-Bereichs folgen.

Die vorstehend aufgeführten Kritikpunkte sollen nicht den Blick dafür verstellen, dass es sich um eine rechtsvergleichend auf dem Boden des geltenden Rechts und nicht um eine rechtspolitisch angelegte Arbeit handelt, was freilich erst der Untertitel erhellt. Wer am deutsch-französischen Rechtsverkehr interessiert ist, wird aus den präzisen und aktuellen Darlegungen zur Rechtslage hinsichtlich der AGBKontrolle in Frankreich (S. $160 \mathrm{ff}$. zum Tatbestand, S. $231 \mathrm{ff}$. zu den Rechtsfolgen) einigen Gewinn ziehen können. Dies gilt nicht zuletzt im Hinblick auf die oben genannte Einschätzung des Autors, dass nach französischem Recht im b2b-Bereich hinsichtlich der AGB-Kontrolle in gewissem Umfang eine weiter reichende Gestaltungsfreiheit besteht als nach deutschem Recht. Zudem beschränkt sich Dorn keineswegs darauf, die Neuregelungen in Art. 1170 und insbesondere Art. 1171 des französischen Code civil darzustellen. Vielmehr vermittelt er auch einen sehr soliden Überblick über das Verständnis, das diese Normen in der französischen Lehre und Praxis erfahren, und über diesbezügliche Kontroversen. Das Werk erweist sich damit zugleich als ein nützliches Instrument zur Erschließung des AGB-Rechts von Frankreich und damit eines der bedeutendsten Versicherungsmärkte.

Funding Open Access funding enabled and organized by Projekt DEAL.

Open Access Dieser Artikel wird unter der Creative Commons Namensnennung 4.0 International Lizenz veröffentlicht, welche die Nutzung, Vervielfältigung, Bearbeitung, Verbreitung und Wiedergabe in jeglichem Medium und Format erlaubt, sofern Sie den/die ursprünglichen Autor(en) und die Quelle ordnungsgemäß nennen, einen Link zur Creative Commons Lizenz beifügen und angeben, ob Änderungen vorgenommen wurden.

Die in diesem Artikel enthaltenen Bilder und sonstiges Drittmaterial unterliegen ebenfalls der genannten Creative Commons Lizenz, sofern sich aus der Abbildungslegende nichts anderes ergibt. Sofern das betreffende Material nicht unter der genannten Creative Commons Lizenz steht und die betreffende Handlung nicht nach gesetzlichen Vorschriften erlaubt ist, ist für die oben aufgeführten Weiterverwendungen des Materials die Einwilligung des jeweiligen Rechteinhabers einzuholen.

Weitere Details zur Lizenz entnehmen Sie bitte der Lizenzinformation auf http://creativecommons.org/ licenses/by/4.0/deed.de. 